\title{
RE-ANALYSIS OF THE RADIO LUMINOSITY FUNCTION OF GALACTIC H II REGIONS
}

\author{
R. Paladini ${ }^{1}$, G. DeZotti ${ }^{2,3}$, A. Noriega-Crespo ${ }^{1}$, and S. J. Carey ${ }^{1}$ \\ ${ }^{1}$ Spitzer Science Center, 1200 East California Boulevard, Pasadena, CA 91125, USA; paladini@ipac.caltech.edu \\ 2 INAF-Osservatorio Astronomico di Padova, Vicolo dell'Osservatorio 5, I-35122 Padova, Italy \\ 3 SISSA, Via Beirut 4, I-24014 Trieste, Italy \\ Received 2008 July 1; accepted 2009 July 14; published 2009 August 18
}

\begin{abstract}
We have re-analyzed continuum and recombination lines radio data available in the literature in order to derive the luminosity function (LF) of Galactic H II regions. The study is performed by considering the first and fourth Galactic quadrants independently. We estimate the completeness level of the sample in the fourth quadrant at $5 \mathrm{Jy}$, and the one in the first quadrant at $2 \mathrm{Jy}$. We show that the two samples (fourth or first quadrant) include, as well as giant and supergiant H II regions, a significant number of subgiant sources. The LF is obtained, in each Galactic quadrant, with a generalized Schmidt's estimator using an effective volume derived from the observed spatial distribution of the considered H II regions. The re-analysis also takes advantage of recently published ancillary absorption data allowing to solve the distance ambiguity for several objects. A single power-law fit to the LFs retrieves a slope equal to $-2.23 \pm 0.07$ (fourth quadrant) and to $-1.85 \pm 0.11$ (first quadrant). We also find marginal evidence of a luminosity break at $L_{\mathrm{knee}}=10^{23.45} \mathrm{erg} \mathrm{s}^{-1} \mathrm{~Hz}^{-1}$ for the LF in the fourth quadrant. We convert radio luminosities into equivalent $\mathrm{H} \alpha$ and Lyman continuum luminosities to facilitate comparisons with extragalactic studies. We obtain an average total H II regions Lyman continuum luminosity of $0.89 \pm 0.23 \times 10^{53} \mathrm{~s}^{-1}$, corresponding to $30 \%$ of the total ionizing luminosity of the Galaxy.

Key words: Galaxy: general - H II regions - radio continuum: stars - stars: luminosity function, mass function
\end{abstract}

\section{INTRODUCTION}

Extensive studies have investigated the luminosity function (LF) of H II regions in external galaxies. These, mostly based on $\mathrm{H} \alpha$ observations, have established that (1) the slope of the LF is correlated with Hubble type, with early-type galaxies having a steeper slope than late-type (Kennicutt et al. 1989, hereafter KEH89); (2) the LF becomes significantly flatter at low luminosities (KEH89; Rand 1992; Walterbos \& Braun 1992; Rozas et al. 1996, 1999, 2000; Bradley et al. 2006). Such observational facts have been interpreted in terms of evolutionary effects (Oey \& Clarke 1998). Variations of the LF in spiral arm versus interarm regions are controversial: some studies claim that the LF of interarm H II regions is steeper (KEH89; Banfi et al. 1993; Rand 1992), but others do not support this conclusion (Rozas et al. 1996; Knapen et al. 1993, Knapen 1998).

Remarkably, there is a surprising paucity of similar studies regarding the $\mathrm{H}$ II regions population of our own Galaxy. This is partly due to the fact that HII regions lie on the plane, where $\mathrm{H} \alpha$ is heavily obscured by dust, therefore limiting the observation to $\mathrm{H}$ II regions located within a radius of $\sim 1 \mathrm{kpc}$. Radio surveys could in principle circumvent this problem but, in the past, only a few have uniformly targeted the Galactic plane region with sufficient angular resolution and sensitivity to resolve individual sources. Likely the near future will see a breakthrough in this direction, with the completion of extensive radio surveys carried out through interferometric techniques, such as the International Galactic Plane Survey (IGPS) at $1.4 \mathrm{GHz}$ and $408 \mathrm{MHz}$, or MAGPIS (White et al. 2005), at 5 and $1.4 \mathrm{GHz}$. Kerton et al. (2007) have published a catalog of Galactic extended sources from the Canadian Galactic Plane survey (Taylor et al. 2002), part of IGPS. However, such a remarkable work of detection and identification of the sources has encompassed the second Galactic quadrant, i.e., the region $90^{\circ}<l<175^{\circ},-3.6<b<5$. 6 .
The reference works for the LF of Galactic H II regions are Smith \& Kennicutt (1989) and McKee \& Williams (1997). They both rely on the list of H II regions compiled by Smith et al. (1978, hereafter SBM). This list is a compilation of $93 \mathrm{H} \mathrm{II}$ regions and is characterized by important caveats. For example, the Southern sources in the list (49 out of 93) are mainly from the $5 \mathrm{GHz}$ recombination lines survey by Wilson et al. (1970). These authors claim that their survey is complete for sources having a peak antenna temperature of $1.3 \mathrm{~K}$, or greater, at $5 \mathrm{GHz}$. However, this statement is based on the continuum data which have been obtained through a variety of surveys, carried out with different frequencies and angular resolutions. For instance, the Thomas \& Day (1969a, 1969b) $2.7 \mathrm{GHz}$ survey has a spatial resolution of 8.2 , while the Hill (1968) $1.4 \mathrm{GHz}$ survey is characterized by a resolving power of $14^{\prime}$. Most importantly, the completeness levels of these continuum surveys appear to be unknown.

Therefore, Wilson et al. cannot be certain that all sources above the $1.3 \mathrm{~K}$ threshold have been picked up, in the first place, by those surveys. This means that the recombination lines survey has an intrinsic bias, with important consequences on the completeness for giant and supergiant $\mathrm{H}$ II regions, as we will discuss in Section 5.

Complementary studies have focused on the LF of ultracompact $\mathrm{H}$ II regions (Comeron \& Torra 1996; Casassus et al. 2000). The work by Comeron \& Torra is probably the only attempt found in the literature to take into account the complex spiral arm geometry of our Galaxy. However, since it relies only on the $I R A S$ point-source catalog (PSC) and lacks any kinematic information on the sources, it purely consists in a multi-parameter fit analysis, which has obvious limitations.

For the reasons described above, we have decided to look in the literature and search for alternative data to the SBM list in order to obtain an independent derivation of the LF of Galactic $\mathrm{H}$ II regions. We are in fact convinced that archival data are widely and unjustifiably underused in this field and, until new 
catalogs of $\mathrm{H}$ II regions are made available, for instance, by the MAGPIS or the IGPS consortia, these archival data should be fully exploited. This is even more true given the new release of ancillary data, such as of those on $\mathrm{H}$ I absorption, which make it possible, for many of these sources, to break, for the first time, the distance degeneracy.

In the following, we will consider two definitions of the LF denoted, respectively, by $n(L) d L$ and $\phi(L) d \log L$. The former gives the number of sources per unit volume with luminosity in the range $[L, L+d L]$ and is usually parameterized as $n(L) d L \propto L^{-\alpha} d L$; the latter gives the number of sources per unit volume with luminosity in the logarithmic interval $[\log L, \log L+d \log L]$ and is represented as $\phi(L) d \log L \propto$ $L^{-\alpha+1} d \log L$.

\section{SAMPLE SELECTION}

The vast majority of cataloged $\mathrm{H}$ II regions is located in the first $\left(0^{\circ}<l<90^{\circ}\right)$ and fourth $\left(270^{\circ}<l<360^{\circ}\right)$ Galactic quadrants.

The most recent, extensive radio recombination line surveys in the Galactic plane are the Caswell \& Haynes (1987, hereafter CH87) survey in the fourth quadrant, and the Lockman (1989) survey in the first quadrant.

The CH87 survey has covered the region $210^{\circ}<l<360^{\circ}$, $|b|<2^{\circ}$, in the fourth quadrant. With an angular resolution of $\sim 4$.'1, it represents the follow up of the continuum $5 \mathrm{GHz}$ survey by Haynes et al. $(1978,1979)$, which has been carried out at the same angular resolution as the recombination lines survey. The CH87 survey consists of $\mathrm{H} 109 \alpha$ and $\mathrm{H} 110 \alpha$ recombination line measurements of most of the sources (as stated by the same authors) with peak brightness temperature exceeding $1 \mathrm{~K}$ at $5 \mathrm{GHz}$, equivalent to a completeness limit, in peak flux density, of $1.3 \mathrm{Jy}$. CH87 provide, for each $\mathrm{H}$ II region in their surveyed region, the measured recombination line velocity and electron temperature, as well as the angular diameter and the integrated flux density as observed in the continuum $5 \mathrm{GHz}$ survey. We point out that these flux densities and diameters have been derived by Haynes et al. by measuring by hand contours of surface brightness. The same method has been applied to the sources in the SBM list located in the southern hemisphere, as described in Wilson et al. (1970).

The Lockman (1989) survey, with a spatial resolution of $3^{\prime}$, is a follow up of the $4.8 \mathrm{GHz}$ Altenhoff et al. (1979) continuum survey, made with comparable angular resolution (2'6). It consists of observations of the $\mathrm{H} 87 \alpha, \mathrm{H} 88 \alpha$, and $\mathrm{H} 85 \alpha$ hydrogen recombination lines, and is complete down to $1 \mathrm{Jy}$, in peak flux density, for $0^{\circ}<l<60^{\circ}$, while for $l>60^{\circ}$, the completeness of the survey is less uniform. Interestingly, the Lockman survey has covered, with equal angular resolution, the same region of the sky of the H110 $\alpha$ and formaldehyde $\left(\mathrm{H}_{2} \mathrm{CO}\right)$ absorption line survey by Downes et al. (1980, hereafter DWBW). DWBW also claim to be complete to a peak flux density of $1 \mathrm{Jy}$, although Lockman reports that $\sim 200$ sources are observed for the first time. This fact could be explained with misidentification problems in the DWBW survey. For the Lockman sources, flux densities and angular diameters are taken from the $4.8 \mathrm{GHz}$ Altenhoff et al. (1979) survey, and these have been measured through automated Gaussian fits. The same technique has been used to derive flux and angular sizes for the sources in the SBM compilations located in the Northern hemisphere, as discussed in Reifenstein et al. (1970).

Instrumental systematics can potentially degrade in a significant way continuum measurements, especially with the tech- nology available 20 or 30 years ago. One of the main problems is represented by $1 / f$ noise which can cause fluctuations of the baselines and make the measured flux densities unreliable. The solution is typically to scan the sky at a fast rate. The scan rates of the Haynes et al.'s and Lockman's surveys are 2.5 minute $^{-1}$ and $80^{\prime}$ minute $^{-1}$, respectively. In contrast, the sources which have been selected by Wilson et al. for their recombination lines survey, have been re-observed in the continuum at $5 \mathrm{GHz}$ by Goss \& Shaver (1970), and these observations provide the base for the flux and diameters quoted in the SBM list. Goss \& Shaver (1970) have scanned the sky at a rate of $1^{\circ}$ minute ${ }^{-1}$, meaning that the uncertainties in the flux densities quoted by $\mathrm{CH} 87$ and Altenhoff et al. (1979), due to effects such as $1 / f$ noise, are lower or at least of the same order as the values reported by SBM.

We note that Paladini et al. (2003, hereafter Paper I) compiled a radio catalog of 1442 Galactic $\mathrm{H}$ II regions for which angular diameters and flux densities at a reference frequency, namely $2.7 \mathrm{GHz}$, are given. This is the so-called Master Catalog, in which, for each source, the quoted values at the reference frequency are obtained by taking the weighted mean over all the available data reported in the literature. This procedure also requires to estimate measurement errors when these are not provided by the authors of the original surveys. For $\sim 800$ of the $\mathrm{H}$ II regions in the catalog, radio recombination lines have been measured and the mean line velocities, line, and electron temperatures are reported in the Master Catalog. Such a data set, combined with the Fich et al. (1989, hereafter FBS89) rotation curve, has been used in Paladini et al. (2004, hereafter Paper II) to compute, for a subset of 575 sources ${ }^{4}$ galactocentric and solar distances.

We could have in principle used the Paladini et al.'s catalog to perform our analysis of the LF. However, as mentioned above, the Master Catalog contains weighted averages of measurements obtained with different instruments, at various frequencies and angular resolutions. This fact per se can introduce complications in, for example, assessing the actual completeness of the sample.

In contrast, the CH87 and Lockman's databases present the advantage (over the Paladini et al.'s catalog but also over the SBM list) of being uniform samples, obtained by observations of large areas of the sky with a single instrument, characterized by a given sensitivity and angular resolution.

\section{COMPLETENESS OF THE SAMPLES}

CH87 observed $316 \mathrm{H}$ II regions in total. The authors state in the paper that they have likely observed most of the sources with $S_{p}>1.3 \mathrm{Jy}$. The first step is then to remove from the sample the sources below this threshold, i.e., since CH87 quote, for each source, the integrated flux at $5 \mathrm{GHz}, S_{i}$, this corresponds to exclude the sources for which

$$
S_{p}=S_{i} \frac{\theta_{b}^{2}}{\theta_{b}^{2}+\theta_{\mathrm{HII}}^{2}} \leqslant 1.3 \mathrm{Jy},
$$

where $\theta_{\mathrm{H} \text { II }}$ is the angular diameter of the source, and $\theta_{b}$ is the angular resolution of the survey (4.4). The application of the above criterion leaves us with $238 \mathrm{H}$ II regions. At this point, we still need to establish the actual completeness of the survey

\footnotetext{
4 Only the sources with a measured velocity $\left|V_{\mathrm{LSR}}\right|>10 \mathrm{~km} \mathrm{~s}^{-1}$ are considered in the analysis.
} 


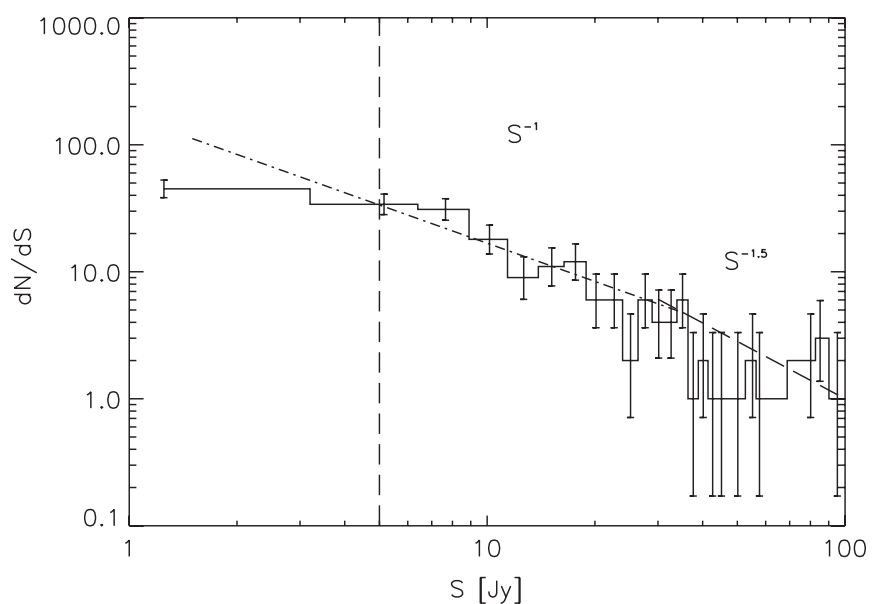

Figure 1. Differential source counts for the CH87 sample. The fits for a disk (dash-dotted line) and spherical (dashed) distributions are shown. The completeness level is denoted by the dashed vertical line.

in terms of flux density. For this purpose, we consider the observed differential counts (see Figure 1) of the sample and we fit to this, along the lines of Paper II, a simple function of the type $S^{-1}$, representing the scaling of the number of sources, as seen from an observer located at the Sun, if these were distributed along a disk. This approximation is valid within the range $30 \mathrm{Jy}>S_{5 \mathrm{GHz}}>5 \mathrm{Jy}$. For $S_{5 \mathrm{GHz}}>30 \mathrm{Jy}$, the distribution steepens and is better represented by a function $S^{-3 / 2}$, appropriate for a uniform three-dimensional distribution, roughly corresponding to nearby sources. Errors have been computed, in each flux bin, by applying the formulae by Gehrels (1986). Specifically, the upper limits are given by

$$
\lambda_{u} \simeq n+S \sqrt{n+\frac{3}{4}}+\frac{S^{2}+3}{4},
$$

while the lower limits are

$$
\lambda_{l} \simeq n\left(1-\frac{1}{9 n}-\frac{S}{3 \sqrt{n}}+\beta n^{\gamma}\right)^{3},
$$

where $S=1, \beta=0$, and $n$ is the number of sources per bin. By removing from the sample the objects with an integrated flux $<5 \mathrm{Jy}$, we are left with 162 sources. In the following, we will derive the LF of the fourth quadrant also for flux cutoffs different (and above) from the cutoff value obtained from the analysis of the differential counts. These additional LFs will be used for comparison with the one obtained for the reference 5 Jy cutoff, in order to estimate the potential error introduced by the assessment of the completeness level for the CH87 sample.

The Lockman (1989) sample has 256 sources within longitude $2^{\circ}<l<60^{\circ}$. After removing the sources with peak flux density $S_{p}<1 \mathrm{Jy}$, or for which

$$
S_{p}=S_{i} \times \frac{\theta_{b}^{2}}{\theta_{b}^{2}+\theta_{\mathrm{H} \text { II }}^{2}} \leqslant 1 \mathrm{Jy}
$$

(taking $\theta_{b}=2$ '.6), we are left with 115 sources. In this case, the source counts are well fitted by a model $\sim S^{-1}$ in the flux range $30 \mathrm{Jy}>S_{5 \mathrm{GHz}} \simeq 2 \mathrm{Jy}$, while for $S_{5 \mathrm{GHz}}>30 \mathrm{Jy}$ a function $S^{-3 / 2}$ is a better representation of the data (see Figure 2). The exclusion of sources below the 2 Jy threshold leaves 93 objects. Along the same lines of the $\mathrm{CH} 87$ sample, we will also compute the LF in the first quadrant for completeness cutoffs above the one estimated from the differential counts.

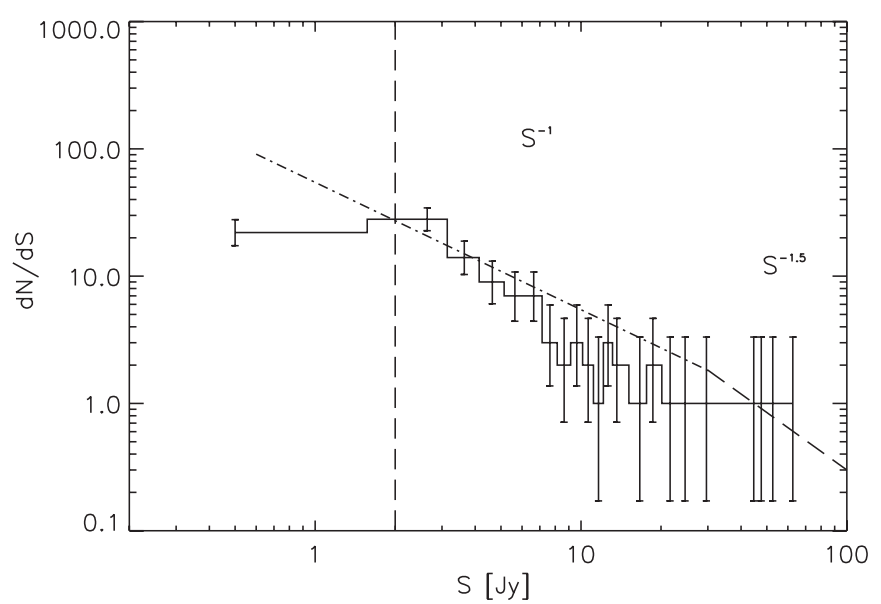

Figure 2. Differential source counts for the Lockman (1989) sample. The fits for a disk (dash-dotted line) and spherical (dashed) distributions are shown. The completeness level is denoted by the dashed vertical line.

\section{EXCLUSION OF SOURCES WITH $\left|V_{\mathrm{LSR}}\right|<10 \mathrm{~km} \mathrm{~s}^{-1}$}

Sources with radial velocities $\left|V_{\mathrm{LSR}}\right|>10 \mathrm{~km} \mathrm{~s}^{-1}$ can, in principle, be affected by noncircular streaming motions, but, as noted for instance by Quireza et al. (2006b), an accurate quantification of this effect is not possible given our current knowledge of the Galactic velocity field. For this reason, throughout the text, we have assumed that the circular motion hypothesis is generally valid.

In contrast, sources with measured radial velocities $<10 \mathrm{~km} \mathrm{~s}^{-1}$ are known to be significantly contaminated by peculiar velocity components, and distances derived by using these velocities can be highly unreliable.

Therefore, from each sample (CH87; Lockman 1989), we remove the sources with $\left|V_{\mathrm{LSR}}\right|<10 \mathrm{~km} \mathrm{~s}^{-1}$. For the reference cases of flux cutoffs of 5 and $2 \mathrm{Jy}$, this corresponds to remove 23 objects in the fourth quadrant and five objects in the first quadrant.

In Section 8.3, we will examine the effect, on the derived LF, due to the removal of the low-velocity sources.

\section{RADIAL AND SOLAR DISTANCES}

For each source in the CH87 and Lockman (1989) samples, we compute the galactocentric and solar distances, $R$ and $D$, respectively, by combining the measured hydrogen recombination line velocity with the rotation curve by FBS89. While in Paper II we used $R_{0}=8.5 \mathrm{kpc}$, we have adopted here a somewhat smaller value, $R_{0}=8 \mathrm{kpc}$, favored by recent determinations (Eisenhauer et al. 2003, 2005). FBS89 provide a useful tabulation of the rotation curve for several values of $R_{0}$ (see Table 4 of their paper), including $R_{0}=8.0 \mathrm{kpc}$. In Section 8.2, we will show that the adoption of rotation curve models other than FBS89 has a very minor impact on the derived LF.

As for the solar distances, part of the H II regions in both samples are affected by the well known distance degeneracy problem. These are the sources within the solar circle for which two solutions are consistent with the observed radial velocity. In this case, we proceed as in Paper II, using auxiliary (optical or absorption) data, when available, to break the degeneracy.

For the fourth quadrant (CH87 sample), with respect to Paper II, we have complemented the list of references of auxiliary data with the work on H I absorption by Fish et al. (2003), Nord et al. (2006), and Quireza et al. (2006a). 


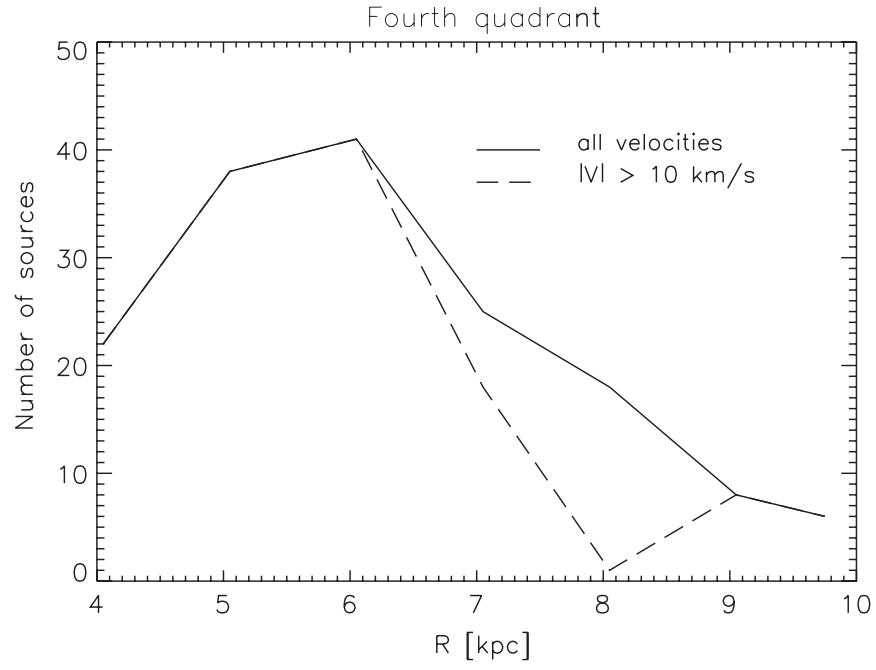

Figure 3. Radial distribution of $\mathrm{H}$ II regions in the fourth Galactic quadrant. Sources are from $\mathrm{CH} 87$, and their flux density is above $5 \mathrm{Jy}$ at $5 \mathrm{GHz}$. Bins of $\Delta R=1 \mathrm{kpc}$. The solid line denotes the distribution obtained when sources with $\left|V_{\mathrm{LSR}}\right|<10 \mathrm{~km} \mathrm{~s}^{-1}$ are left in the sample, while the dashed line corresponds to the case when these sources are removed.

When sources lack auxiliary data, we make use of the luminosity-physical diameter correlation found in Paper II and, in particular, of Equation (7) in that paper, which, for the $5 \mathrm{GHz}$ case takes the form

$$
D=C \times 10^{\frac{a}{2-b}}\left(\frac{\theta}{1^{\prime}}\right)^{\frac{b}{2-b}}\left(\frac{v}{1 \mathrm{~Hz}}\right)^{\frac{-1}{2-b}}\left(\frac{S}{1 \mathrm{Jy}}\right)^{\frac{-1}{2-b}} \mathrm{kpc},
$$

with $C=1.2767 \times 10^{-19}, a=32.4$, and $b=0.86 .{ }^{5}$ For the reference case of a flux cutoff at $5 \mathrm{Jy}$, we have applied Equation (5) to 61 out of 139 sources, corresponding to $44 \%$ of the total.

We emphasize that the above relation is used to choose between the near and far kinematic solutions for a given measured line velocity, not to compute a heliocentric distance from the observed angular diameter and flux. Following Paper II, we performed on the $\mathrm{CH} 87$ sample of sources a test to cross check whether the application of Equation (5) to the sources with auxiliary data assigns the correct solution. The test shows that the luminosity-diameter correlation allows us to choose the correct solution in $\sim 80 \%$ of the cases.

In the first quadrant, 79 out of 88 of the Lockman sources above the reference flux cutoff at $2 \mathrm{Jy}$ lie inside the solar circle. The first step is to make use of auxiliary (optical and absorption) data to solve the kinematic distance ambiguity. As for the CH87, we complement the list of references of Paper II with the H I absorption data by Fish et al. (2003), Kolpak et al. (2003), Quireza et al. (2006b), Anderson \& Bania (2009), as well as with the formaldehyde $\left(\mathrm{H}_{2} \mathrm{CO}\right)$ absorption data provided by Araya et al. (2002), Watson et al. (2003), Sewilo et al. (2004), and the same DWBW. However, as noted by Anderson \& Bania, $\mathrm{HI}$ absorption methods are usually more robust than $\mathrm{H}_{2} \mathrm{CO}$ to break the distance degeneracy, given that $\mathrm{H}$ I is more ubiquitous than $\mathrm{H}_{2} \mathrm{CO}$. For this reason, whenever a source has both $\mathrm{H}_{2} \mathrm{CO}$ and $\mathrm{HI}$ absorption data, we favor the solution associated with H I. Following these criteria, we are able to solve the distance ambiguity for 70 objects. To the remaining nine (10\% of the total) sources, we have applied Equation (5).

\footnotetext{
5 Note that, in Paladini et al. (2004), Equation (7) is missing the factor $1.2767 \times 10^{-19}$ and the frequency $v$ is in wrong units, i.e., $\mathrm{GHz}$ instead of $\mathrm{Hz}$.
}

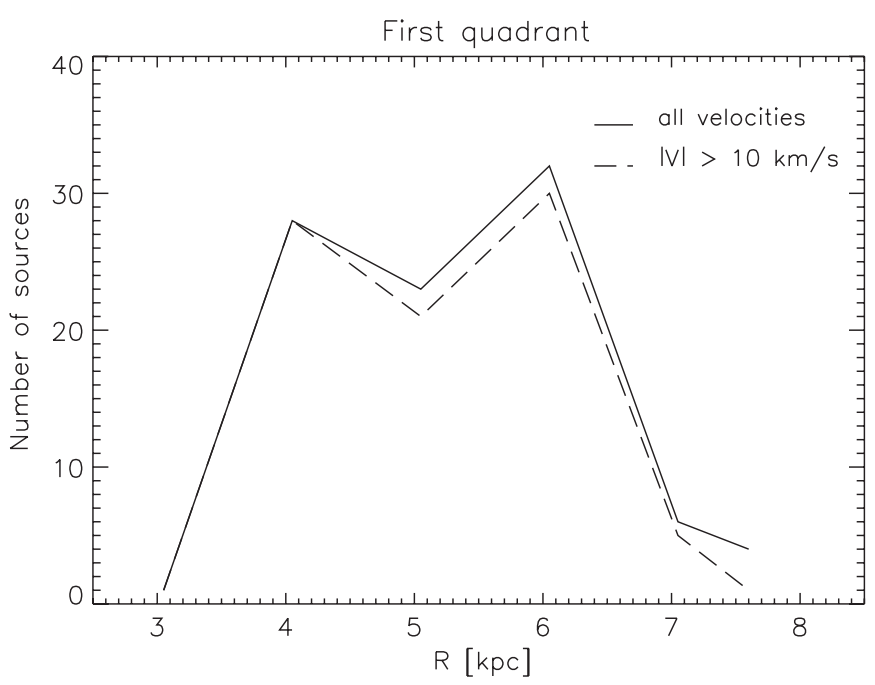

Figure 4. Radial distribution of $\mathrm{H}$ II regions in the first Galactic quadrant. Sources are from Lockman (1989), and their flux density is above $2 \mathrm{Jy}$ at $4.8 \mathrm{GHz}$. Bins of $\Delta R=1 \mathrm{kpc}$. As for the fourth quadrant, the solid line denotes the distribution obtained by including all sources; the dashed line denotes the one corresponding to the removal of sources with $\left|V_{\mathrm{LSR}}\right|<10 \mathrm{~km} \mathrm{~s}^{-1}$.

The radial distributions of the $\mathrm{H}$ II regions above the flux cutoffs of 5 and 2 Jy are illustrated in Figures 3 and 4, for the fourth and first quadrant, respectively. Note that the figures show the radial distribution both when sources with $\left|V_{\mathrm{LSR}}\right|<10 \mathrm{~km} \mathrm{~s}^{-1}$ are removed or left in the samples. Clearly, the removal of these sources causes, in the fourth quadrant, an artificial dip in the distribution for $R \sim 8 \mathrm{kpc}$.

Remarkably, and regardless of the exclusion of the lowvelocity sources, the radial distribution appears to be different in the two quadrants. As emphasized by several authors (e.g., Lockman 1981, 1989; CH87), this is a consequence of the fact that $\mathrm{H}_{\mathrm{II}}$ regions, as massive stars in general, trace the spiral structure of the Galaxy and, as such, reflect in their spatial distribution the asymmetry of the arms geometry as seen by an observer located at the Sun position.

\section{COMPOSITION OF THE SAMPLES}

The SBM compilation used in Smith \& Kennicutt (1989) and McKee \& Williams (1997) is supposedly complete, over the whole Galaxy, for supergiant $\mathrm{H}$ II regions, and for our side of the Galaxy, for giant $\mathrm{H}$ II regions. SBM define supergiant $\mathrm{H}$ II regions those having a Lyman continuum luminosity $N_{c}>50 \times$ $10^{49} \mathrm{~s}^{-1}$, while giant $\mathrm{H}_{\mathrm{II}}$ regions are the ones for which $4 \times$ $10^{49} \mathrm{~s}^{-1}>N_{c}>50 \times 10^{49} \mathrm{~s}^{-1}$. These definitions are provided taking as a reference the luminosity of Orion for $R_{0}=10 \mathrm{kpc}$ and $T_{e}=10,000 \mathrm{~K}$. Since in our derivation of the radial and solar distances, we use $R_{0}=8 \mathrm{kpc}$ and $T_{e} \sim 7000 \mathrm{~K}$, we rescale, following McKee \& Williams, the Lyman luminosities by a factor of 0.75 which takes into account both corrections. Accordingly, the new limits for supergiant and giant $\mathrm{H}$ II regions become, respectively, $N_{c}>37.5 \times 10^{49} \mathrm{~s}^{-1}$ and $3 \times 10^{49} \mathrm{~s}^{-1}>$ $N_{c}>37.5 \times 10^{49} \mathrm{~s}^{-1}$. In addition, subgiant $\mathrm{H}_{\text {II }}$ regions will be the sources with an ionizing luminosity less than $3 \times 10^{49} \mathrm{~s}^{-1}$.

We use the flux densities tabulated by Haynes et al. (1978) for the CH87 sources and those by Altenhoff et al. (1979) for the Lockman sample, combined with the solar distances assigned as described in the previous sections, to compute for each $\mathrm{H}_{\mathrm{II}}$ region in the fourth and first quadrants its ionizing luminosity, following the Condon's (1992) relation 
Table 1

Composition of CH87 and Lockman Samples

\begin{tabular}{lcc}
\hline \hline \multicolumn{1}{c}{ Type } & CH87 & Lockman (1989) \\
\hline Subgiant H II regions & 74 & 51 \\
Giant H II regions & 62 & 35 \\
Supergiant H II regions & 3 & 2 \\
\hline
\end{tabular}

Note. Quoted values are for the reference cases of a flux cutoff at 5 and $2 \mathrm{Jy}$, for the CH87 and Lockman (1989) samples, respectively.

$$
\begin{aligned}
N_{c}= & 6.3 \times 10^{52} \text { photons s}^{-1}\left(\frac{T_{e}}{10^{4} \mathrm{~K}}\right)^{-0.45}\left(\frac{v}{\mathrm{GHz}}\right)^{0.1} \\
& \times \frac{L_{v}}{10^{27} \mathrm{erg} \mathrm{s}^{-1} \mathrm{~Hz}^{-1}},
\end{aligned}
$$

where, for the electron temperature, we use $T_{e}=6200 \mathrm{~K}$ for the sources in the fourth quadrant, and $T_{e}=7400 \mathrm{~K}$ for the objects in the first quadrant. These numbers represent mean values of the electron temperatures of the sources in the $\mathrm{CH} 87$ and Lockman's samples.

Applying the definition of subgiant, giant, and supergiant H II regions given above, we obtain the composition of the $\mathrm{CH} 87$ and Lockman samples provided in Table 1.

Clearly, in both quadrants we have a significant contribution from subgiant $\mathrm{H}$ II regions. This is important given that, as noted by McKee \& Williams (1997), most of the ionizing luminosity of the Galaxy is likely contributed by small H II regions, rather than by the supergiant and giant populations. Furthermore, we note that, for the fourth quadrant, the SBM list quotes 49 between giant and supergiant $\mathrm{H}$ II regions, while the CH87 sample includes 65 in total. Likewise, in the first quadrant, the SBM compilation has 33 giant and supergiant H II regions in the region $2^{\circ}<l<60^{\circ}$, compared to 37 for the Lockman (1989) sample. This shows that the samples considered here are likely more complete than the SBM catalog, as mentioned in the Introduction.

\section{THE LF FOR AN INHOMOGENEOUS SOURCE DISTRIBUTION}

One of the limitations of previous works which have investigated the LF for Galactic $\mathrm{H}$ II regions is that they did not allow for a realistic geometric distribution of the sources. In this section, we describe the approach adopted for our analysis. Under the assumption that the LF is independent of position, the number $N(L)$ of $\mathrm{H}$ II regions with luminosity $L$ within $d \log L$ detected above a flux threshold $S$ is related to the LF, $\phi(L) d \log L$, by

$$
\begin{aligned}
N(L) d \log L= & \phi(L) d \log L \int_{0}^{D_{\max }(S \mid L)} d D D \\
& \times \int_{l_{\min }}^{l_{\max }} d l n(R) \int_{z_{\min }}^{z_{\max }} d z \rho(z),
\end{aligned}
$$

with $R=\left(R_{0}^{2}+D^{2}-2 D R_{0} \cos l\right)^{1 / 2}$. In the above expression, $n(R)$ and $\rho(z)$ describe the radial and vertical density profiles, respectively, and the multiple integral on the right-hand side is the effective volume within which a source of luminosity $L$ is included in the catalog, i.e.,

$$
\begin{aligned}
V_{\mathrm{eff}}(L \mid S)= & \int_{0}^{D_{\max }(S \mid L)} d D D \\
& \times \int_{l_{\min }}^{l_{\max }} d l n(R) \int_{z_{\min }}^{z_{\max }} d z \rho(z),
\end{aligned}
$$

where $D_{\max }(S \mid L)=(L / 4 \pi S)^{1 / 2}, z_{\max }=D \sin \left(b_{\max }\right) \simeq$ $D b_{\max }$, and $z_{\min }=D \sin \left(b_{\min }\right) \simeq D b_{\min }$.

From Equations (7) and (8), we can define a generalized Schmidt's (1968) estimator for the LF:

$$
\phi(L) \Delta \log L=\sum_{\mathrm{i}} \frac{1}{V_{\mathrm{eff}, \mathrm{i}}(L \mid S)},
$$

where the sum is over all sources with luminosity within the interval $\Delta \log L$ around $L$. In Equation (8), for the vertical density profile we use a function of the form

$$
\rho(z \mid R)=\exp \left[-0.5\left(z / \sigma_{z}\right)^{2}\right]
$$

If $z_{\max }$ is much larger than the vertical scale length $\sigma_{z}$ and $z_{\min }=-z_{\max }$, the integral over $d z$ on the right-hand side of Equation (8) gives $(2 \pi)^{1 / 2} \sigma_{z}$.

Bronfman et al. (2000) have investigated the scale height distribution of a population of 748 OB stars, for which (their Table 2) they tabulate values at different Galactocentric radii, for the north and south hemispheres, both separately and combined. We have fitted their quoted values for the combined distribution, after correcting for $R_{0}=8 \mathrm{kpc}$. The data result to be well represented by a function of the form

$$
z_{1 / 2}=2.85 R^{1.69}
$$

where $z_{1 / 2}=(2 \ln 2)^{1 / 2} \sigma_{z}$. This relation is also consistent with the range of values ( 39 to $52 \mathrm{pc}$ ) quoted in Paper II for H II regions in our Galaxy, therefore we adopt it.

The choice of the radial density profile is more complex (see Figure 12 of Paper II). In Section 5, we have derived the distribution in Galactocentric distances of the sources in our samples located in the fourth (Figure 3) and first (Figure 4) quadrants. Based on these distributions, we model the $n(R)$ as

$$
\begin{aligned}
n(R)= & \exp \left[-\left(\left(R-R_{\mathrm{pk} 1}\right) / \sigma_{r 1}\right)^{2}\right] \\
& +A \exp \left[-\left(\left(R-R_{\mathrm{pk} 2}\right) / \sigma_{r 2}\right)^{2}\right] .
\end{aligned}
$$

In the above expressions, $R_{\mathrm{pk} 1}, R_{\mathrm{pk} 2}, \sigma_{r 1}, \sigma_{r 2}$, and $A$ are free parameters to be determined fitting the observed distribution of sources as a function of the galactocentric distance $R$, given by

$$
\begin{aligned}
\mathcal{N}(R) \Delta R= & n(R) R \Delta R \int_{\theta_{\min }(R)}^{\theta \max (R)} d \theta \\
& \cdot \int_{z_{\min }(R, \theta)}^{z_{\max }(R, \theta)} d z \rho(z, R) \int_{\log L_{\min }(R, \theta)}^{\log L_{\max }} d \log L \phi(L) .
\end{aligned}
$$

We note that the adoption of a double-Gaussian profile for the first quadrant is motivated by the fact that the line of sight intercepts the Carina and Scutum-Crux spiral arms.

To keep the number of parameters within a manageable limit, we adopt, in Equation (13), the usual power-law representation for the luminosity function $\phi(L) d \log L \propto L^{-1} d \log L$ (i.e., $\alpha=2$ ), after having checked that the results are little affected by changes of its slope within the currently accepted range, $1.5 \leqslant \alpha \leqslant 2.5$. The estimate of $\phi(L)$ is thus based on an iterative approach: we use a first guess on the shape of $\phi(L)$ to derive the parameters for $n(R)$ that we then exploit to obtain a better estimate for the LF. More details on the calculation are provided in the Appendix. 


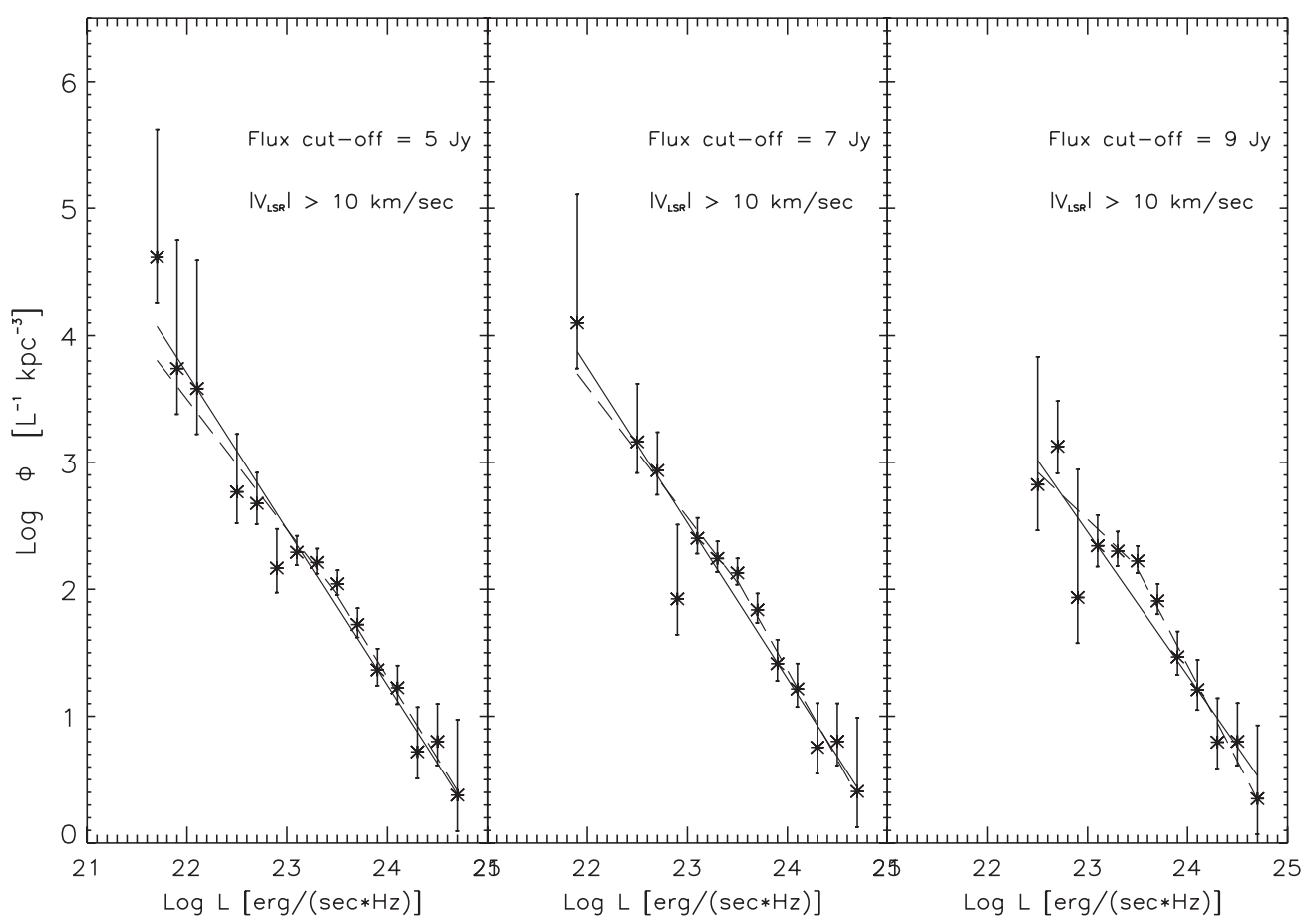

Figure 5. LFs in the fourth quadrant for different completeness levels. The solid line indicates the best-fit LF. Also shown (dashed line) is a two-component power-law fit.

Table 2

Best-fit Parameters of the Radial Profile for the Fourth Quadrant

\begin{tabular}{lccc}
\hline \hline Parameter & $5 \mathrm{Jy}$ & $7 \mathrm{Jy}$ & $9 \mathrm{Jy}$ \\
\hline$R_{\mathrm{pk} 1}$ & $4.28(\mathrm{kpc})$ & $4.26(\mathrm{kpc})$ & $4.27(\mathrm{kpc})$ \\
$R_{\mathrm{pk} 2}$ & $9.54(\mathrm{kpc})$ & $9.53(\mathrm{kpc})$ & $9.53(\mathrm{kpc})$ \\
$\sigma_{r 1}$ & $1.44(\mathrm{kpc})$ & $1.45(\mathrm{kpc})$ & $1.45(\mathrm{kpc})$ \\
$\sigma_{r 2}$ & $0.42(\mathrm{kpc})$ & $0.42(\mathrm{kpc})$ & $0.42(\mathrm{kpc})$ \\
$A$ & 0.30 & 0.29 & 0.30 \\
\hline
\end{tabular}

Table 3

Best-fit Parameters of the Radial Profile for the First Quadrant

\begin{tabular}{lccc}
\hline \hline Parameter & $2 \mathrm{Jy}$ & $3 \mathrm{Jy}$ & $4 \mathrm{Jy}$ \\
\hline$R_{\mathrm{pk} 1}$ & $5.17(\mathrm{kpc})$ & $5.17(\mathrm{kpc})$ & $5.16(\mathrm{kpc})$ \\
$R_{\mathrm{pk} 2}$ & $3.70(\mathrm{kpc})$ & $3.71(\mathrm{kpc})$ & $3.69(\mathrm{kpc})$ \\
$\sigma_{r 1}$ & $0.83(\mathrm{kpc})$ & $0.82(\mathrm{kpc})$ & $0.82(\mathrm{kpc})$ \\
$\sigma_{r 2}$ & $0.39 \mathrm{kpc}$ & $0.39(\mathrm{kpc})$ & $0.41(\mathrm{kpc})$ \\
$A$ & 6.50 & 6.51 & 6.68 \\
\hline
\end{tabular}

The minimum $\chi^{2}$ values of the parameters for both samples are listed in Tables 2 and 3. In view of a derivation of an LF for different levels of completeness, we have derived the bestfit parameters for the radial distribution for each value of flux cutoff. For the reference cases of flux cutoffs at 5 and 2 Jy for the CH87 and Lockman's samples, we obtain that the best fits correspond to a $\chi_{r}^{2}$ of 2.08 and 1.13 , for a bin size $\Delta R=1 \mathrm{kpc}$. The value of $\chi_{r}^{2}$ is actually an overestimate and does not denote an unacceptably poor fit because the adopted errors correspond only to the Poisson fluctuations of the number of sources in each bin and do not include the contributions from the uncertainties in the estimates of distances $R$. Thus, although we are well aware that the axially symmetric distributions of Equation (12) are certainly an oversimplification, a more refined model, entailing more parameters, does not appear to be warranted in the present data situation.

\section{THE LF IN THE FOURTH AND FIRST QUADRANT}

The LFs for the CH87 and Lockman's samples derived by using Equation (9) and the radial profile $n(R)$ determined in the previous section are shown in Figures 5 and 6 . The bin size is $\Delta \log L=0.2$. This choice makes it possible to perform direct comparisons with LFs obtained, with the same binning, by other authors (e.g., Smith \& Kennicutt 1989; KEH89).

The errors in each bin are computed by applying the formulae by Gehrels (1986; see Section 3). In this case, the effective number of sources per bin, $n_{\mathrm{eff}}$, is given by

$$
n_{\mathrm{eff}}=\frac{\left(\sum_{i} \frac{1}{V_{\mathrm{effi} i}}\right)^{2}}{\sum_{i}\left(\frac{1}{V_{\mathrm{effi} i}}\right)^{2}} .
$$

A single power-law fit to the data retrieves the slopes provided, for each quadrant and flux cutoff, in Tables 4 and 5. Quoted errors are obtained from the fitting procedure and are only indicative of the actual errors. We have also tried to fit the data with a two-component power law of the form

$$
\phi(L)=A\left\{\begin{array}{ll}
\left(L / L_{\text {knee }}\right)^{-\alpha^{\prime}+1} & \text { if } L<L_{\text {knee }} \\
\left(L / L_{\text {knee }}\right)^{-\beta^{\prime}+1} & \text { if } L>L_{\text {knee }}
\end{array} .\right.
$$

From Tables 6 and 7, we see that, for the CH87 sample, since $\Delta \chi^{2} \geqslant 2.3$ (see, e.g., Bevington \& Robinson 1992), the addition of two parameters in the fit is justified and the two-component power-law model appears to be favored for all completeness levels, while for the Lockman (1989) sample, the single powerlaw model always corresponds to the best $\chi^{2}$ value.

To facilitate comparisons with published LFs, we convert our monochromatic LF (in units of erg s $\mathrm{s}^{-1} \mathrm{~Hz}^{-1}$ ) into Lyman continuum fluxes, $N_{c}$ (in photons $\mathrm{s}^{-1}$ ) and into $\mathrm{H} \alpha$ luminosities, $L(\mathrm{H} \alpha)$, in erg $\mathrm{s}^{-1}$. To convert into Lyman continuum fluxes, we make use of Equation (6). 


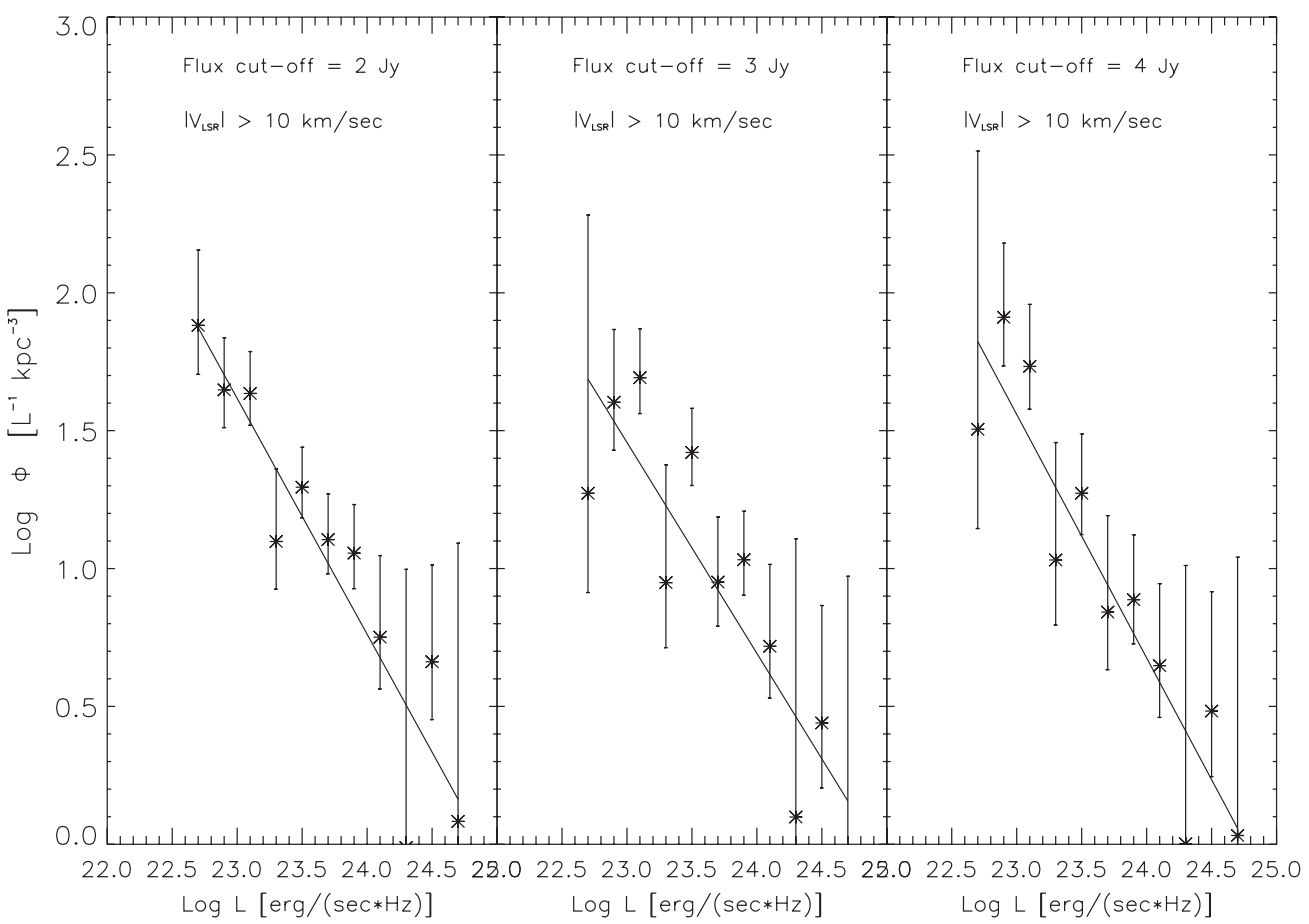

Figure 6. LFs in the first quadrant for different completeness levels. The solid line indicates the best-fit LF.

Table 4

Best-fit Values for the LFs in the Fourth Quadrant for Different Levels of Completeness (Single Power Law)

\begin{tabular}{lccc}
\hline \hline Parameter & $5 \mathrm{Jy}$ & $7 \mathrm{Jy}$ & $9 \mathrm{Jy}$ \\
\hline$\alpha$ & $2.23 \pm 0.07$ & $2.22 \pm 0.09$ & $2.13 \pm 0.12$ \\
$\chi^{2}$ & 19.49 & 17.34 & 27.81 \\
$\chi_{r}^{2}$ & 1.49 & 1.58 & 2.78 \\
\hline
\end{tabular}

Table 5

Best-fit Values for the LFs in the First Quadrant for Different Levels of Completeness (Single Power Law)

\begin{tabular}{lccc}
\hline \hline Parameter & $2 \mathrm{Jy}$ & $3 \mathrm{Jy}$ & $4 \mathrm{Jy}$ \\
\hline$\alpha$ & $1.85 \pm 0.11$ & $1.76 \pm 0.14$ & $1.88 \pm 0.12$ \\
$\chi^{2}$ & 11.97 & 23.02 & 11.53 \\
$\chi_{r}^{2}$ & 1.33 & 2.56 & 1.28 \\
\hline
\end{tabular}

Table 6

Best-fit Values for the LFs in the Fourth Quadrant for Different Levels of Completeness (Two-component Power Law)

\begin{tabular}{lccc}
\hline \hline Parameter & $5 \mathrm{Jy}$ & $7 \mathrm{Jy}$ & $9 \mathrm{Jy}$ \\
\hline$\alpha^{\prime}$ & $2.02 \pm 0.11$ & $2.03 \pm 0.15$ & $1.74 \pm 0.22$ \\
$\beta^{\prime}$ & $2.27 \pm 0.19$ & $2.41 \pm 0.20$ & $2.51 \pm 0.20$ \\
$\log L_{\text {knee }}$ & $23.45 \pm 0.11$ & $23.54 \pm 0.08$ & $23.47 \pm 0.12$ \\
$\log A$ & 2.13 & 2.19 & 2.04 \\
$\chi^{2}$ & 13.43 & 9.88 & 8.69 \\
$\chi_{r}^{2}$ & 1.22 & 1.09 & 1.08 \\
\hline
\end{tabular}

To express the LF in terms of $\mathrm{H} \alpha$ luminosities, we use the relation (Osterbrock 1974)

$$
\frac{L(\mathrm{H} \beta) / h \nu_{\mathrm{H} \beta}}{N_{c}} \sim \frac{\alpha_{\mathrm{H} \beta}^{\mathrm{eff}}\left(T_{e}\right)}{\alpha_{\beta}\left(T_{e}\right)},
$$

which implies that the number of photons emitted by the nebula in $\mathrm{H} \beta$ is directly proportional to its Lyman continuum
Table 7

Best-fit Values for the LFs in the First Quadrant for Different Levels of Completeness (Two-component Power Law)

\begin{tabular}{lccc}
\hline \hline Parameter & $2 \mathrm{Jy}$ & $3 \mathrm{Jy}$ & $4 \mathrm{Jy}$ \\
\hline$\alpha^{\prime}$ & $1.71 \pm 0.12$ & $1.68 \pm 0.14$ & $1.96 \pm 0.15$ \\
$\beta^{\prime}$ & $1.98 \pm 0.33$ & $2.22 \pm 0.35$ & $1.93 \pm 0.37$ \\
$\log L_{\text {knee }}$ & $24.05 \pm 0.23$ & $23.94 \pm 0.45$ & $23.11 \pm 0.53$ \\
$\log A$ & 1.75 & 1.84 & 1.88 \\
$\chi^{2}$ & 15.47 & 21.47 & 14.24 \\
$\chi_{r}^{2}$ & 2.21 & 3.07 & 2.03 \\
\hline
\end{tabular}

flux. The hydrogen recombination coefficients $\alpha_{\mathrm{H} \beta}^{\text {eff }}$ and $\alpha_{\beta}$ are tabulated, for various electron densities and temperatures, by Osterbrock (1974) and Storey \& Hummer (1995). By assuming an electron density $n_{e}=10^{2} \mathrm{~cm}^{-3}$, we derive the recombination coefficients at $T_{e}=6200 \mathrm{~K}(\mathrm{CH} 87)$ and $T_{e}=7400 \mathrm{~K}$ (Lockman 1989) by interpolation over the tabulated values. We adopt the value of $4.08 \mathrm{eV}$ for the energy of a photon emitted in a transition between quantum states $n=4$ and $n=2$. The $\mathrm{H} \alpha$ luminosities are then obtained from $L(\mathrm{H} \beta)$ by interpolating at $6200 \mathrm{~K}$ and $7400 \mathrm{~K}$ the Balmer decrements compiled by Osterbrock (1974) for different values of $T_{e}$ (Figures 7 and 8).

\subsection{Effect of the Choice of the Radial Density Profile}

In this subsection, we discuss how the LF determined through the application of Equation (9) depends on the adopted radial density profile. To illustrate the effect of $n(R)$, we compare the LFs obtained for the CH87 sample by using three different functional forms, namely, the radial profile of Equation (12) with the best-fit values for the parameters in Table 2, an exponential profile, a constant profile. The reference case for a flux cutoff at $5 \mathrm{Jy}$ is considered. We chose the exponential radial profile derived by Kent et al. (1991) from the analysis of the Infrared Telescope (IRT) $2.4 \mu \mathrm{m}$ data

$$
n(R)=\exp \left(-R / R_{d}\right)
$$




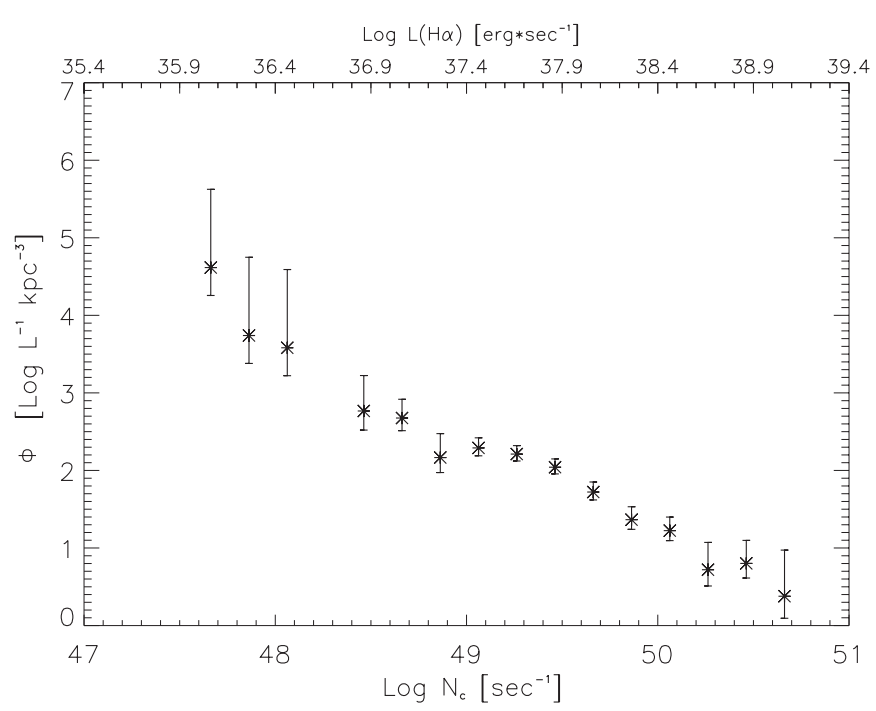

Figure 7. LF in the fourth quadrant expressed in units of Lyman continuum flux (lower scale) and $\mathrm{H} \alpha$ luminosity (upper scale). Results refer to a $5 \mathrm{Jy}$ flux cutoff.

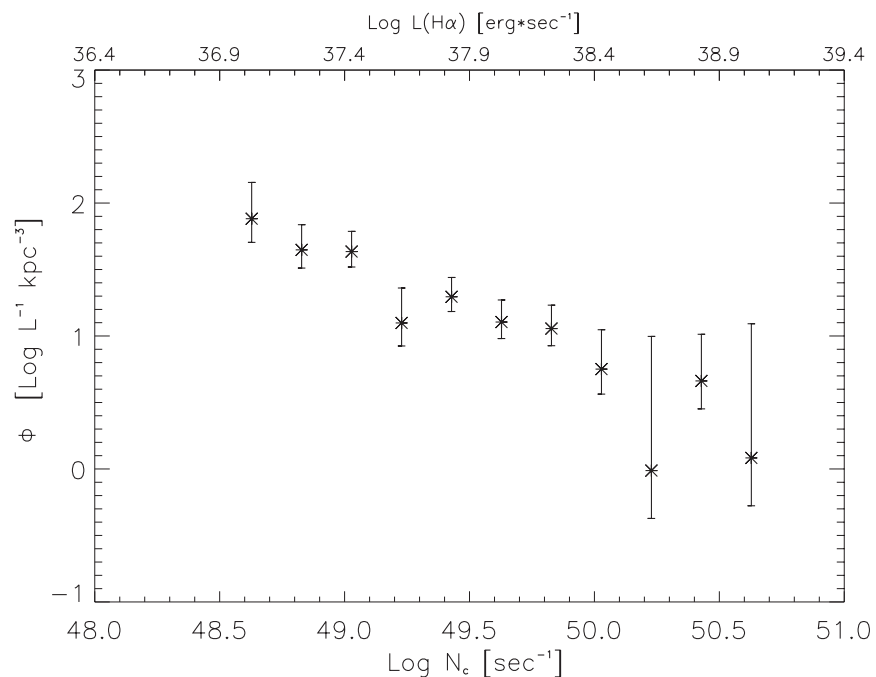

Figure 8. $\mathrm{LF}$ in the first quadrant expressed in units of Lyman continuum flux (lower scale) and $\mathrm{H} \alpha$ luminosity (upper scale). Results refer to a 2 Jy flux cutoff.

with $R_{d}=3 \mathrm{kpc}$. The above expression is used here only for illustrative purposes, therefore a discussion regarding the exact value of the radial scale length, $R_{d}$, is beyond the scope of the present paper. In the case of a constant profile, we use the mean of the values tabulated by Bronfman et al. (2000) for the surface density of OB stars in annuli of increasing Galactocentric radius (see Table 2 of their paper)

$$
n(R)=1 \text { source } \mathrm{kpc}^{-2} \text {. }
$$

Once again, the value of the constant is only indicative. Figure 9 shows the LFs resulting from using, alternatively, a double-Gaussian (Equation (12)), an exponential (Equation (17)), and a uniform (Equation (18)) radial profile. Table 8 provides additional information, i.e., the best-fit values of the parameters (power-law index and break luminosity) for each curve. We have used a two-component power-law model given that, in the previous section, we have shown that it better represents the data for the $\mathrm{CH} 87$ sample.

Clearly, the choice of the density profile affects the estimate of the LF, although the derived values of the parameters are consistent with each other, within the errors.

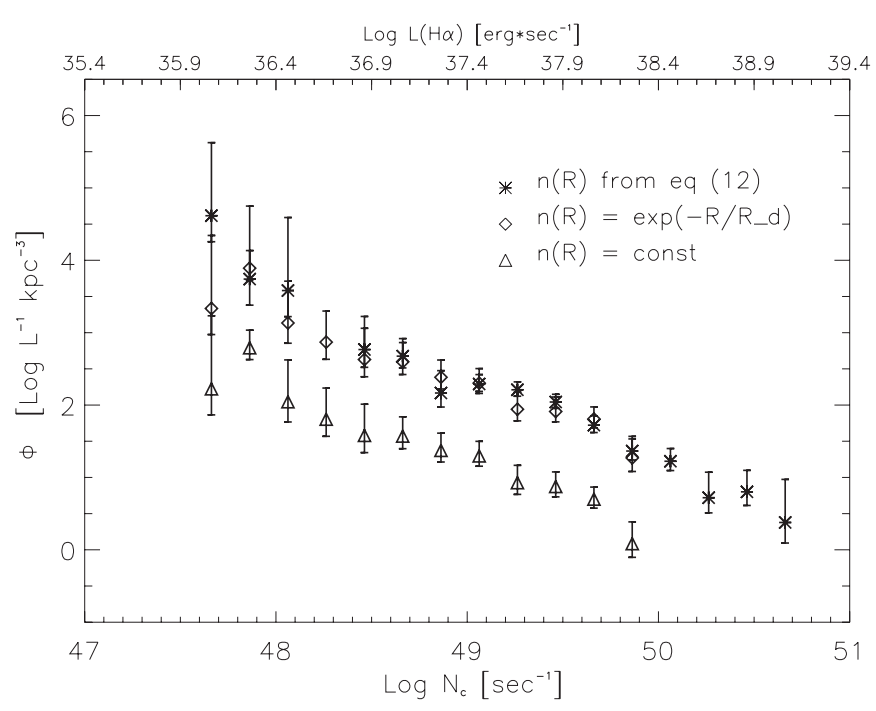

Figure 9. LF for the CH87 sample (5 Jy flux cutoff) obtained by adopting: a double-Gaussian radial profile (Equation (12), stars), an exponential profile (diamonds), and a constant profile (triangles).

\section{Table 8}

Best-fit Values Parameters of the LFs for the Fourth Quadrant (5 Jy Flux Cutoff) Obtained with Different Radial Density Profiles

\begin{tabular}{lllc}
\hline \hline \multicolumn{1}{c}{$n(R)$} & $\alpha^{\prime}\left(L<L_{\text {knee }}\right)$ & $\beta^{\prime}\left(L>L_{\text {knee }}\right)$ & $\log L_{\text {knee }}$ \\
\hline Equation $(12)$ & $2.19 \pm 0.20$ & $2.46 \pm 0.0 .15$ & $23.45 \pm 0.11$ \\
Exponential & $1.99 \pm 0.19$ & $2.06 \pm 0.42$ & $23.47 \pm 0.09$ \\
Constant & $1.92 \pm 0.12$ & $2.98 \pm 0.63$ & $23.33 \pm 0.15$ \\
\hline
\end{tabular}

Note. Fit for a two-component power-law LF (Equation (15)).

The different normalizations for the three cases reflect the different effective volumes associated with each density profile. This is also clearly illustrated by Equation (7), where one can see that the effective volume acts as a normalization constant, while the shape of the LF is determined by the observed quantity $N(L) d \log L$.

\subsection{Effect of the Choice of the Rotation Curve}

In principle, the LF derived in Section 8 could be dependent on the choice of the rotation curve model applied to the data to convert the measured line velocities into Galactocentric and solar distances. As discussed in Section 5, our adopted rotation curve model is the one by FBS89. To illustrate the possible dependence of our results on this choice, we recomputed the LF for the CH87 sample by using two alternative models, i.e., the rotation curve by Clemens (1985) and the one by Brand \& Blitz (1993). The comparison between the LFs obtained by adopting these models with the one derived by using FBS89 is shown in Figure 10. Clearly, the three LFs almost overlap with each other, showing that our results are not sensitive to different choices for the rotation curve.

\subsection{Effect of the Inclusion of Sources with $\left|V_{\mathrm{LSR}}\right|<10 \mathrm{~km} \mathrm{~s}^{-1}$}

In this section, we investigate how the removal of sources with $\left|V_{\mathrm{LSR}}\right|<10 \mathrm{~km} \mathrm{~s}^{-1}$ affects the derived LFs. For this purpose, we recompute, for each sample and considered completeness level, the effective volume, after examination of the radial distribution of the sources. These are illustrated in Figures 3 and 4. As noted in Section 5, the removal of the low-velocity sources in the fourth quadrant causes a dip in the distribution for $R \sim 8 \mathrm{kpc}$. 


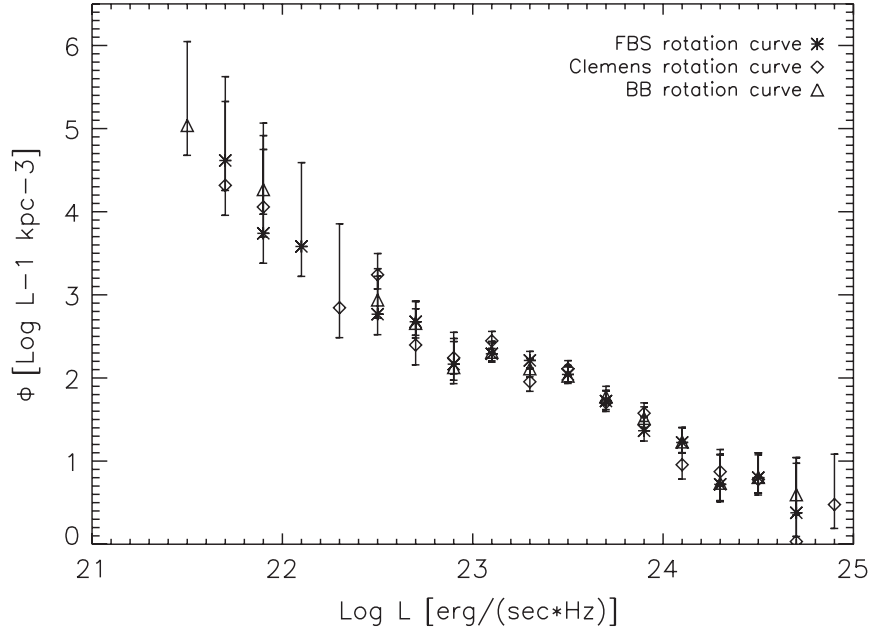

Figure 10. Comparison between LF obtained for the $\mathrm{CH} 87$ sample by adopting different rotation curves. A flux cutoff of $5 \mathrm{Jy}$ is adopted.

For this reason, instead of applying Equation (12) to fit for $n(R)$, we use a Gaussian profile with inner cutoff, of the form

$$
\begin{aligned}
n_{4 \mathrm{th}}(R)= & \exp \left[-\left(\left(R-R_{\mathrm{pk}}\right) / \sigma_{r}\right)^{2}\right] \\
& \times \exp \left[-\left(\left(R-R_{\mathrm{int}}\right) / R\right)\right] .
\end{aligned}
$$

For the Lockman (1989) sample, as in Section 7, we adopt Equation (12), since the addition of the sources with $\left|V_{\mathrm{LSR}}\right|<$ $10 \mathrm{~km} \mathrm{~s}^{-1}$ leaves the radial distribution basically unchanged. For illustrative purpose, we provide the best-fit parameters for the radial profile (Table 9), as well as the slopes (Table 10) of the LFs (Figure 10), for the fourth quadrant only, given that the $\mathrm{CH} 87$ sample is more impacted by the presence of sources with low measured velocities. From inspection of Table 10 and Figure 10 and comparison with Table 4 and
Table 9

Best-fit Parameters of the Radial Profile for the Fourth Quadrant

\begin{tabular}{lccc}
\hline \hline Parameter & $5 \mathrm{Jy}$ & $7 \mathrm{Jy}$ & $9 \mathrm{Jy}$ \\
\hline$R_{\mathrm{pk}}$ & $3.21(\mathrm{kpc})$ & $2.82(\mathrm{kpc})$ & $3.13(\mathrm{kpc})$ \\
$R_{\text {int }}$ & $1.94(\mathrm{kpc})$ & $2.08(\mathrm{kpc})$ & $1.99(\mathrm{kpc})$ \\
$\sigma_{r}$ & $2.85(\mathrm{kpc})$ & $3.24(\mathrm{kpc})$ & $3.09(\mathrm{kpc})$ \\
\hline
\end{tabular}

Note. All sources (including those for which $\left|V_{\mathrm{LSR}}\right|<10 \mathrm{~km} \mathrm{~s}^{-1}$ ) are considered.

Table 10

Best-fit Values for the LFs in the Fourth Quadrant for Different Levels of Completeness (Single Power Law)

\begin{tabular}{lccc}
\hline \hline Parameter & $5 \mathrm{Jy}$ & $7 \mathrm{Jy}$ & $9 \mathrm{Jy}$ \\
\hline$\alpha$ & $2.09 \pm 0.06$ & $2.14 \pm 0.06$ & $2.17 \pm 0.08$ \\
$\chi^{2}$ & 26.96 & 20.55 & 22.40 \\
$\chi_{r}^{2}$ & 1.58 & 1.21 & 1.49 \\
\hline
\end{tabular}

Note. Sources with $\left|V_{\mathrm{LSR}}\right|<10 \mathrm{~km} \mathrm{~s}^{-1}$ are also included.

Figure 5, we see that the slopes are consistent with the values retrieved by removing from the sample the low-velocity sources. In the case considered here, though, we find no evidence for a luminosity knee (see Figure 11). However, such a result should be interpreted with caution, due to the large uncertainty associated with the sources with $\left|V_{\mathrm{LSR}}\right|<10 \mathrm{~km} \mathrm{~s}^{-1}$.

\section{DISCUSSION}

In the following, we will refer, unless stated otherwise, to the reference cases of a flux cutoff at $5 \mathrm{Jy}$ for the $\mathrm{CH} 87$ sample, and at $2 \mathrm{Jy}$ for the Lockman (1989) sample. In addition, we consider the case for which sources with $\left|V_{\mathrm{LSR}}\right|<10 \mathrm{~km} \mathrm{~s}^{-1}$ have been removed from the samples.

In the fourth quadrant, the $\mathrm{H} \alpha$ luminosities of the $\mathrm{CH} 87$ sample span $\sim 3$ orders of magnitude, from $10^{36}$ to $10^{39} \mathrm{erg} \mathrm{s}^{-1}$

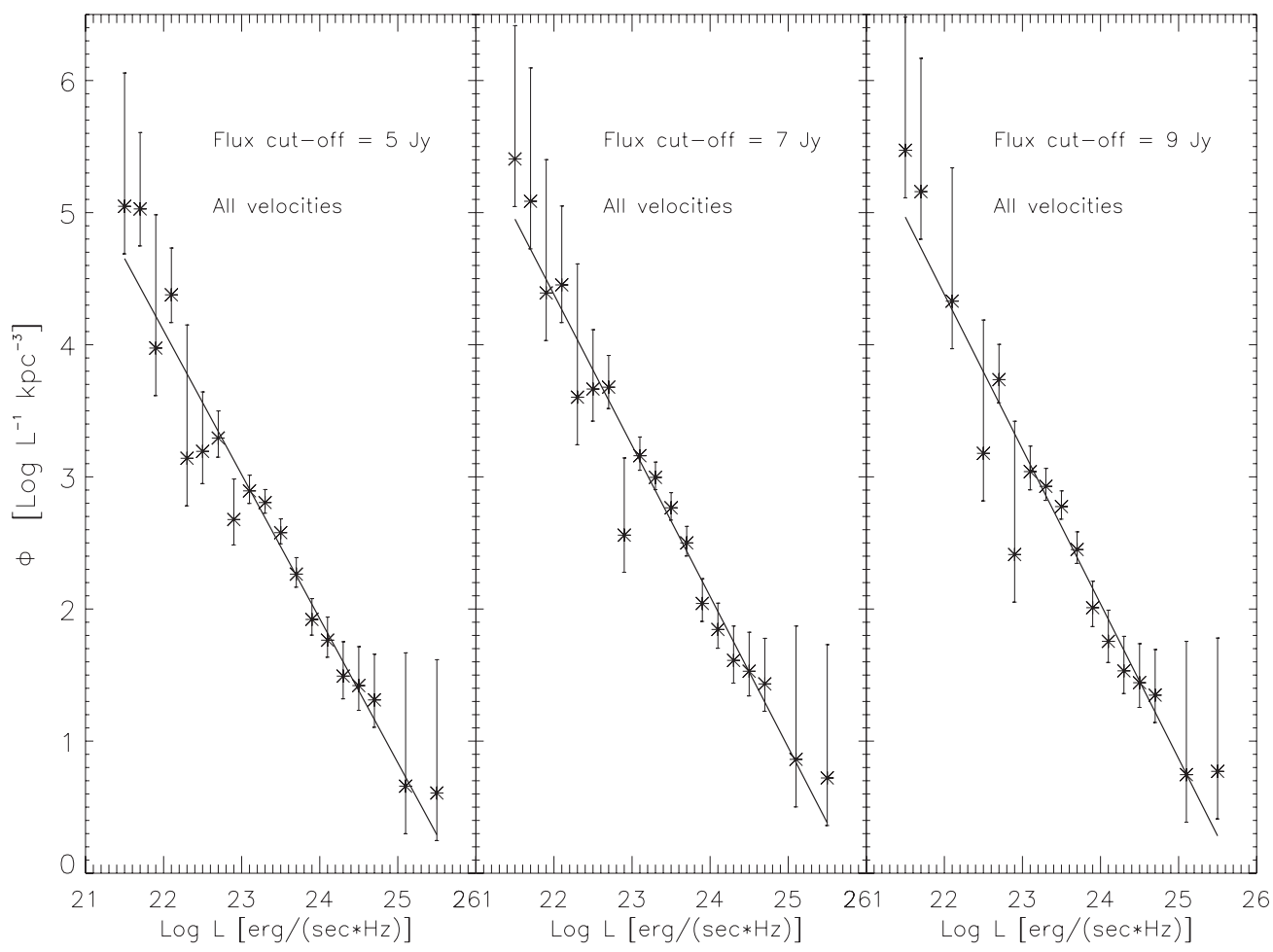

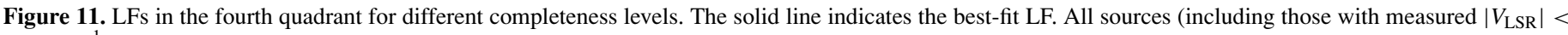
$10 \mathrm{~km} \mathrm{~s}^{-1}$ ) are considered. 
or, in equivalent Lyman continuum luminosities, from $10^{47.6}$ to $10^{50.6}$ photons $\mathrm{s}^{-1}$. The lower limit corresponds to $\mathrm{H}$ II regions ionized by a single star, while the upper value is indicative of the lack, in our Galaxy, of supergiant $\mathrm{H}$ II regions such as 30 Doradus in the Large Magellanic Clouds, which originate from very large $\mathrm{OB}$ associations.

The LF derived from the CH87 sample presents marginal evidence for a change of slope at $L_{\text {knee }} \sim 10^{23.45} \mathrm{erg} \mathrm{s}^{-1} \mathrm{~Hz}^{-1}$, corresponding to an $\mathrm{H} \alpha$ luminosity of $\sim 10^{37.75} \mathrm{erg} \mathrm{s}^{-1}$ and to a Lyman continuum luminosity of $\sim 10^{49.41}$ photons $\mathrm{s}^{-1}$ (Figure 7, upper and lower scales). A break in the LF of H II regions has been observed by many authors in external galaxies. Among these, KEH89 were the first to conduct a systematic study of the $\mathrm{H}$ II region LF in 30 spiral and irregular galaxies. For six galaxies (of Hubble type Sab-Sb) of their sample, they find that the LF has an abrupt turnover at $\log L(\mathrm{H} \alpha)=38.7-$ 39.0. Recently, Bradley et al. (2006) have reported a mean value for the break luminosity of $\log L(\mathrm{H} \alpha)=38.6 \pm 0.1$, based on a sample of 56 spiral galaxies. Such a break has been interpreted in several ways. KEH89 suggest that the change in the slope corresponds to a transition between normal $\mathrm{H}$ II regions (ionized by a single star or by a small association) and the class of supergiants. Oey \& Clarke (1998) seem to favor the hypothesis that the break might be caused by evolutionary effects and a maximum number of stars per cluster, while Beckman et al. (2000) argue that it is due to the fact that, at luminosities above a given threshold, density bounding prevails on ionization bounding.

As for the slope of the LF, our findings are in agreement with published estimates from extragalactic studies as well as with results obtained for our own Galaxy. For instance, KEH89 report, for Sbc-Sc galaxies, a power-law index in the range $1.5-2.5,^{6}$ while McKee \& Williams (1997) and Smith $\&$ Kennicutt (1989) find, respectively, $\alpha=1.99 \pm 0.25$ and $\alpha=2.3 \pm 0.5$ by fitting Lyman continuum luminosities greater than $10^{49.5}$ photons $\mathrm{s}^{-1}$ and using the SMB sample. These values are both consistent with the slope obtained for a single power-law fit, $\alpha=2.23 \pm 0.07$, as well as with $\beta^{\prime}=2.27 \pm 0.19$ that we quote in Section 7 for $L>L_{\text {knee }}$, in the case of a double power law.

In the first quadrant, the $\mathrm{H} \alpha$ luminosities (or the equivalent Lyman continuum luminosities) of the Lockman (1989) span a smaller range of values than the $\mathrm{CH} 87$ data set, i.e., from $10^{37}$ to $10^{39} \mathrm{erg} \mathrm{s}^{-1}$ or, in equivalent Lyman continuum luminosities, from $10^{48.6}$ to $10^{50.6}$ photons $\mathrm{s}^{-1}$. In this case, the $\mathrm{LF}$ is significantly flatter than in the fourth quadrant, suggesting a possible intrinsic difference between the $\mathrm{H}$ II regions population in the two quadrants, as also hinted by the discrepancy in the radial profiles (see Section 4.).

The LF obtained for the fourth and first quadrants were used to estimate the total ionizing luminosity of Galactic H II regions, given by

$$
L_{\mathrm{tot}}=V_{\mathrm{GP}} \int_{L_{\min }}^{L_{\mathrm{max}}} n(L) L d L,
$$

where $n(L)=\phi(L) /(L \ln 10)$ and $V_{\mathrm{GP}}$ is the volume of the Galactic plane occupied by classical H II regions, i.e.,

$$
V_{\mathrm{GP}}=4 \pi \int_{0}^{R_{\max }} n(R) R d R \int_{0}^{\infty} \rho(z) d z,
$$

with $n(R)$ given by Equation (12) and the best-fit parameters in Tables 2 and 3, and $\rho(z)$ as in Equation (10). In addition,

\footnotetext{
6 Retrieved from single power-law fits.
}

Table 11

Total Luminosities Derived from the LFs in the Fourth Quadrant

\begin{tabular}{lccc}
\multicolumn{1}{c}{ Derived Quantity } & $5 \mathrm{Jy}$ & $7 \mathrm{Jy}$ & $9 \mathrm{Jy}$ \\
\hline$V_{\mathrm{GP}}\left(\mathrm{kpc}^{3}\right)$ & 8.98 & 8.85 & 8.99 \\
$L_{\mathrm{Gal}, 5 \mathrm{GHz}}\left(\mathrm{erg} \mathrm{s}^{-1} \mathrm{~Hz}\right)$ & $0.95 \times 10^{27}$ & $1.30 \times 10^{27}$ & $0.63 \times 10^{27}$ \\
$L_{\mathrm{Gal}, N_{c}}\left(\mathrm{~s}^{-1}\right)$ & $0.87 \times 10^{53}$ & $1.19 \times 10^{53}$ & $0.58 \times 10^{53}$ \\
$L_{\mathrm{Gal}, \mathrm{H} \alpha}\left(\mathrm{erg} \mathrm{s}^{-1}\right)$ & $0.19 \times 10^{42}$ & $0.26 \times 10^{42}$ & $0.13 \times 10^{42}$
\end{tabular}

Table 12

Total Luminosities Derived from the LFs in the First Quadrant

\begin{tabular}{lccc}
\hline \hline \multicolumn{1}{c}{ Derived Quantity } & $2 \mathrm{Jy}$ & $3 \mathrm{Jy}$ & $4 \mathrm{Jy}$ \\
\hline$V_{\mathrm{GP}}\left(\mathrm{kpc}^{3}\right)$ & 10.49 & 10.77 & 10.45 \\
$L_{\mathrm{Gal}, 5 \mathrm{GHz}}\left(\mathrm{erg} \mathrm{s}^{-1} \mathrm{~Hz}\right)$ & $1.11 \times 10^{27}$ & $0.82 \times 10^{27}$ & $1.31 \times 10^{27}$ \\
$L_{\mathrm{Gal}, N_{c}}\left(\mathrm{~s}^{-1}\right)$ & $0.94 \times 10^{53}$ & $0.70 \times 10^{53}$ & $1.11 \times 10^{53}$ \\
$L_{\mathrm{Gal}, \mathrm{H} \alpha}\left(\mathrm{erg} \mathrm{s}^{-1}\right)$ & $0.21 \times 10^{42}$ & $0.15 \times 10^{42}$ & $0.24 \times 10^{42}$ \\
\hline
\end{tabular}

$R_{\max }=15 \mathrm{kpc}$, and, for the fourth quadrant, $L_{\min }=10^{21.8} \mathrm{erg}$ $\mathrm{s}^{-1} \mathrm{~Hz}, L_{\max }=10^{25} \mathrm{erg} \mathrm{s}^{-1} \mathrm{~Hz}$, while, for the first quadrant, $L_{\min }=10^{22.5} \mathrm{erg} \mathrm{s}^{-1} \mathrm{~Hz}, L_{\max }=10^{24.5}$ (see Figures 5 and 6). The total luminosities obtained by the application of Equation (20) are shown in Tables 11 and 12. Also provided are the values derived for flux completeness levels above the reference cutoff. We note that our estimates are lower limits to the actual total luminosities, given that Equation (20) is integrated between a limited luminosity range. From the values quoted in Tables 11 and 12, the average of the fourth and first quadrants is $(0.89 \pm 0.23) \times 10^{53} \mathrm{~s}^{-1}$.

The total $\mathrm{H}$ II regions Lyman continuum flux for the reference cutoffs can be compared to the total ionizing flux of the Galaxy. Bennett et al. (1994) find $N_{c}=3.5 \times 10^{53} \mathrm{~s}^{-1}$, based on COBE observations of the [N II] $205 \mu \mathrm{m}$ line, while McKee $\&$ Williams (1997) quote a value of $N_{c}=2.56 \times 10^{53} \mathrm{~s}^{-1}$. Recently, Murray \& Rahman (2009), from the analysis of WMAP data, have estimated $N_{c}=3.2 \times 10^{53} \mathrm{~s}^{-1}$. Remarkably, these numbers account for the combined contributions of individual $\mathrm{H}$ II regions and the diffuse warm ionized gas (WIM). In particular, H II regions are likely responsible for only a fraction (between $10 \%$ and $30 \%$ ) of the total emission, as found, among other authors, in our analysis of the $5 \mathrm{GHz}$ Galactic plane free-free emission (Paladini et al. 2005). Of the $\mathrm{H}$ II regions contribution, a significant amount is supposed to originate in subgiant, or small $\mathrm{H}$ II regions, as noted, for instance, by McKee \& Williams (1997). In particular, giant, and subgiant H II regions are likely to contribute $20 \%$ of the total ionizing luminosity of the Galaxy, while another $\sim 20 \%$ is supposedly due to small H II regions (see their Table 3). McKee \& Williams (1997) find a total ionizing luminosity from giant and supergiant $\mathrm{H}$ II regions of $0.57 \times 10^{53} \mathrm{~s}^{-1}$ which, scaled by a factor accounting for the difference in the adopted $R_{0}$ value ( 8.5 in their case), becomes equal to $0.50 \times 10^{53} \mathrm{~s}^{-1}$. Taking into account the contribution from small $\mathrm{HII}$ regions, this number needs to be doubled, to roughly $1 \times 10^{53} \mathrm{~s}^{-1}$, i.e., one-third of the total Lyman continuum flux of the Galaxy, consistent with our results.

\section{CONCLUSIONS}

We have investigated the LF of Galactic H II regions in the fourth and first quadrants, using the samples from the CH87 recombination lines survey for the fourth quadrant, and from the Lockman (1989) recombination lines survey for the first quadrant. We have shown that a good determination of the 
highly inhomogeneous spatial distribution of $\mathrm{HII}$ regions is essential to obtain an accurate estimate of the LF: commonly used but inaccurate density profiles affect both the shape and the normalization of the LF.

The LF in the fourth quadrant is well represented by a single power law with spectral index $\alpha=2.23 \pm 0.07$, although we find that $\chi^{2}$ goodness-of-fit analysis slightly favors a two-component power law with a break luminosity at $\log \left(L(\mathrm{H} \alpha) / \mathrm{erg} \mathrm{s}^{-1}\right)=37.75$. The LF in the first quadrant appears to be flatter, and the best fit is obtained in this case for a single power law with slope $\alpha=1.85 \pm 0.11$.

The inferred total contribution of individual $\mathrm{H}$ II regions to the global ionizing luminosity of the Galaxy is found to be in agreement with the estimates by McKee \& Williams (1997) and roughly equal to one third.

Support for this work was provided by NASA through an award issued by JPL/Caltech. G.D.Z. acknowledges support by ASI contracts I/016/07/0 “COFIS," Planck LFI Activity of Phase E2, and the Spitzer Space Telescope Enhanced Science Program. The comments by the anonymous referee have lead us to discover a serious numerical error in the previous version of the paper and have helped to improve it in many respects.

\section{APPENDIX}

From Equation $R=\left(R_{0}^{2}+D^{2}-2 D R_{0} \cos l\right)^{1 / 2}$, we obtain

$$
D=R_{0} \cos (l) \pm\left(R^{2}-R_{0}^{2} \sin ^{2}(l)\right)^{1 / 2} .
$$

If we call $\theta$ the angle between the direction from the Galactic center (GC) to the Sun and the direction from the GC to the source (i.e., between $R_{0}$ and $R$ ), the sine theorem gives

$$
\sin (\theta)=\frac{D}{R} \sin (l) .
$$

Combining these equations, we get

$$
\sin (\theta)=\left[\frac{R_{0}}{R} \cos (l) \pm\left(1-\left(\frac{R_{0}}{R}\right)^{2} \sin ^{2}(l)\right)^{1 / 2}\right] \sin (l)
$$

The number of sources detected at distance $R$, within $\Delta R$, from the GC can be written as

$$
\begin{aligned}
\mathcal{N}(R) \Delta R= & n(R) R \Delta R \int_{\theta_{\min }(R)}^{\theta \max (R)} d \theta \int_{z_{\min }(R, \theta)}^{z_{\max }(R, \theta)} d z \rho(z, R) \\
& \times \int_{\log L_{\min }(R, \theta)}^{\log L_{\max }} d \log L \phi(L)
\end{aligned}
$$

where $z_{\max , \min }(R, \theta)=b_{\max , \min } D(R, \theta)$ and $L_{\min }(R, \theta)=$ $4 \pi D^{2}(R, \theta) S_{\text {lim }}$, with $D(R, \theta)$ given by

$$
D=\left(R_{0}^{2}+R^{2}-2 R R_{0} \cos \theta\right)^{1 / 2} .
$$

If we have a sample selected in the longitude range $l_{\min } \leqslant l \leqslant$ $l_{\max }$ and $R \leqslant R_{0} \sin l_{\max }$ (i.e., $R$ is smaller than the minimum distance between the GC and the line at $l=l_{\max }$, assuming $l<90^{\circ}$ ), we have

$$
\begin{aligned}
\sin \theta_{\max , \min }(R)= & \left\{\frac{R_{0}}{R} \cos l_{\min } \pm\left[1-\left(\frac{R_{0}}{R}\right)^{2} \sin ^{2} l_{\min }\right]^{1 / 2}\right\} \\
& \times \sin l_{\min }
\end{aligned}
$$

where $\theta_{\max }$ obviously corresponds to the + sign. Note that $\theta_{\max }>\pi / 2$, so that $\theta_{\max }=\pi-\arcsin \left(\sin \theta_{\max }\right)$, while $\theta_{\min }=\arcsin \left(\sin \theta_{\min }\right)$.

If $R>R_{0} \sin l_{\max }$, we do not have contributions to $\mathcal{N}(R)$ from the range $\theta_{1} \leqslant \theta \leqslant \theta_{2}$, given by

$$
\begin{aligned}
\sin \theta_{2,1}(R)= & \left\{\frac{R_{0}}{R} \cos l_{\max } \pm\left[1-\left(\frac{R_{0}}{R}\right)^{2} \sin ^{2} l_{\max }\right]^{1 / 2}\right\} \\
& \times \sin l_{\max } .
\end{aligned}
$$

In practice, we split the integral over $\theta$ in two parts, from $\theta_{\min }$ to $\theta_{1}$ and from $\theta_{2}$ to $\theta_{\max }$, where $\theta_{2,1}$ are given by Equation (A7) if $R>R_{0} \sin l_{\max }$ or by $\theta_{1}=\theta_{2}=\pi / 2-l_{\max }$ otherwise.

\section{REFERENCES}

Altenhoff, W. J., Downes, D., Pauls, T., \& Schraml, J. 1979, A\&A, 35, 23

Anderson, L. D., \& Bania, T. M. 2009, ApJ, 690, 706

Araya, E., et al. 2002, ApJS, 138, 63

Banfi, M., Rampazzo, R., Chincarini, G., \& Henry, R. B. C. 1993, A\&A, 280, 373

Beckman, J. E., Rozas, M., Zurita, A., Watson, R. A., \& Knapen, J. H. 2000, AJ, 119,2728

Bennett, C. L., et al. 1994, ApJ, 434, 587

Bevington, P. R., \& Robinson, D. K. 1992, Data Reduction and Error Analysis for the Physical Sciences (New York: McGraw-Hill)

Bradley, T. R., Knapen, J. H., Beckman, J. E., \& Folkes, S. L. 2006, A\&A, 459 13

Brand, J., \& Blitz, L. 1993, A\&A, 275, 67

Bronfman, L., Casassus, S., May, J., \& Nyman, L.-A. 2000, A\&A, 358, 521

Casassus, S., Bronfman, L., May, J., \& Nyman, L.-A. 2000, A\&A, 358, 514

Caswell, J. L., \& Haynes, R. F. 1987, A\&A, 171, 261

Clemens, D. 1985, ApJ, 295, 422

Comeron, F., \& Torra, J. 1996, A\&A, 314, 776

Condon, J. J. 1992, ARA\&A, 30, 575

Downes, D., Wilson, T. L., Bieging, J., \& Wink, J. 1980, A\&AS, 40, 379

Eisenhauer, F., et al. 2003, ApJ, 597, 121

Eisenhauer, F., et al. 2005, ApJ, 628, 246

Fich, M., Blitz, L., \& Stark, A. A. 1989, ApJ, 342, 272

Fish, V. L., et al. 2003, ApJ, 587, 701

Gehrels, N. 1986, ApJ, 303, 336

Goss, W. M., \& Shaver, P. A. 1970, Aust. J. Phys. Astrophys. Suppl., 14, 1

Haynes, R. F., Caswell, J. L., \& Simons, L. W. J. 1978, Aust. J. Phys. Astrophys. Suppl., 45, 1

Haynes, R. F., Caswell, J. L., \& Simons, L. W. J. 1979, Aust. J. Phys. Astrophys. Suppl., 48, 1

Hill, E. R. 1968, Aust. J. Phys., 21, 735

Kennicutt, R. C., Edgar, B. K., \& Hodge, P. W. 1989, ApJ, 337, 761

Kent, S. M., Dame, T. M., \& Fazio, G. 1991, AJ, 378, 131

Kerton, C. R., Murphy, J., \& Patterson, J. 2007, MNRAS, 379, 289

Knapen, J. H. 1998, MNRAS, 297, 255

Knapen, J. H., Arnth-Jensen, N., Cepa, J., \& Beckman, J. E. 1993, AJ, 106, 56

Kolpak, M., et al. 2003, ApJ, 582, 756

Lockman, F. J. 1981, ApJ, 245, 459

Lockman, F. J. 1989, ApJS, 71, 469

McKee, C. F., \& Williams, J. P. 1997, ApJ, 476, 144

Murray, N., \& Rahman, M. 2009, arXiv:0906.1026

Nord, M. E., et al. 2006, AJ, 132, 242

Oey, M. S., \& Clarke, C. J. 1998, AJ, 115, 1543

Osterbrock, D. E. 1974, Astrophysics of Gaseous Nebulae (San Francisco, CA: Freeman)

Paladini, R., Davies, R. D., \& De Zotti, G. 2004, MNRAS, 347, 237

Paladini, R., deZotti, G., Davies, R. D., \& Giard, M. 2005, MNRAS, 360, 1545

Paladini, R., et al. 2003, A\&A, 397, 213

Quireza, C., Rood, R. T., Balser, D. S., \& Bania, T. M. 2006a, ApJS, 165, 338

Quireza, C., et al. 2006b, ApJ, 653, 1226

Rand, R. J. 1992, AJ, 103, 815

Reifenstein, E. C., III, et al. 1970, A\&A, 4, 357

Rozas, M., Beckman, J. E., \& Knapen, J. H. 1996, A\&A, 307, 735

Rozas, M., Zurita, A., \& Beckman, J. E. 2000, A\&A, 354, 823

Rozas, M., Zurita, A., Heller, C. H., \& Beckman, J. E. 1999, A\&AS, 135, 145 
Schmidt, M. 1968, ApJ, 151, 393

Sewilo, M., et al. 2004, ApJ, 154, 553

Smith, T. R., Biermann, P., \& Mezger, P. G. 1978, A\&A, 66, 65

Smith, T. R., \& Kennicutt, R. 1989, PASP, 101, 649

Storey, P. J., \& Hummer, D. G. 1995, MNRAS, 272, 41

Taylor, A. R., et al. 2002, AJ, 125, 314

Thomas, B. M., \& Day, G. A. 1969a, Aust. J. Phys. Astrophys. Suppl., 11, 3
Thomas, B. M., \& Day, G. A. 1969b, Aust. J. Phys. Astrophys. Suppl., 11,19

Walterbos, R. A. M., \& Braun, R. 1992, A\&AS, 92, 625

Watson, C., et al. 2003, ApJ, 587, 714

White, R. L., Becker, R. H., \& Helfand, D. J. 2005, AJ, 130, 586

Wilson, T. L., Mezger, P. G., Gardner, F. F., \& Milne, D. K. 1970, A\&A, 6, 364 